\title{
TREATMENT OF DEPRESSION WITH CLINICAL OR PSYCHIATRIC COMORBIDITIES: RECOMMENDATIONS FROM HIGH-QUALITY CLINICAL PRACTICE GUIDELINES
}

\author{
F. Gabriel ${ }^{1,5}$, R. Fraguas Jr. ${ }^{2,4}$, C. G. R. C. Molino ${ }^{1,5}$, N. C. L. Santos ${ }^{1,5}$, R. O. Valentim ${ }^{1,5}$, R. A. M. Silva ${ }^{3,5}$, D. O. Melo ${ }^{3,5}$, E. \\ Ribeiro ${ }^{1,4,5}$. \\ ${ }^{1}$ Faculdade de Ciências Farmacêuticas da Universidade de São Paulo, São Paulo, Brasil. \\ ${ }^{2}$ Faculdade de Medicina da Universidade de São Paulo, São Paulo, Brasil. \\ ${ }^{3}$ Instituto de Ciências Ambientais, Químicas e Farmacêuticas da Universidade Federal de São Paulo, São Paulo, Brasil. \\ ${ }^{4}$ Hospital Universitário da Universidade de São Paulo, São Paulo, Brasil. \\ ${ }^{5}$ Chronic Diseases and Informed Decisions (Chronide) Group
}

OBJECTIVES: To create a matrix of recommendations from high-quality Clinical Practice Guidelines (CPGs) for the pharmacological treatment of depression with clinical or psychiatric comorbidities.

\section{BACKGROUND:}

Clinical and psychiatric comorbidities may restrict or decrease the response to the treatment of depression.

High-quality CPGs should provide the best clinical evidence for patient care and optimize healthcare resources, and thus, they should also consider clinical or psychiatric comorbidities.

MATERIALS AND METHODS:

CPGs for the pharmacological treatment of depression in adults, published in English, Portuguese, or Spanish, from January 2011 to August 2017, were systematically searched on Medline (via Pubmed), Embase, the Cochrane Library, and 12 specific CPGs databases (as seen in Molino et al., 2019). CPGs were considered high quality if they scored $\geq 80 \%$ in the Development rigor domain of the Appraisal of Guidelines for
Research \& Evaluation (AGREE) II instrument. A matrix of recommendations was created based on the selected CPGs as well as on the Canadian Network for Mood and Anxiety Treatments (CAMNAT) and the American Psychiatric Association (APA), which were CPGs included for their widespread use in clinical practice. All stages had at least two examiners, and disagreement between them was solved by discussion with a third examiner.

\section{RESULTS AND CONCLUSIONS:}

The six CPGs, except CAMNAT and APA, discussed only anxiety, insomnia, and dementia (comorbidities; Table 1). Recommendations for depression with anxiety and insomnia were the most frequently discussed, including benzodiazepine and hypnotic indication.

High-quality CPGs for depression essentially discussed only anxiety, insomnia, and dementia as comorbidities. Thus, future CPGs should include recommendations for other comorbidities and clinical conditions, thereby providing more information for patient care in depression treatment.

Table 1: Clinical practice guidelines (CPGs) for the treatment of depression with clinical or psychiatric comorbidities

\begin{tabular}{|c|c|c|c|c|c|c|}
\hline Comorbidities $\quad$ CPGs & Chilean & Colombian & England & ICSI & APA & CAMNAT \\
\hline Anxiety and insomnia & & $x$ & $x$ & & $x$ & $x$ \\
\hline Dementia & & $x$ & $x$ & & $x$ & $x$ \\
\hline Obesity & & & & & $x$ & \\
\hline Breast cancer & & & & & $x$ & \\
\hline Diabetes & & & & & $x$ & \\
\hline Cognitive dysfunction & & & & & & $x$ \\
\hline Chronic pain & & & & & $x$ & $\mathrm{X}$ \\
\hline Hypertension & & & & & $x$ & \\
\hline Parkinson's disease & & & & & $x$ & \\
\hline
\end{tabular}

